\title{
Estimating age at first maturity in fish from change-points in growth rate
}

\author{
Robert D. Scott ${ }^{1,2, *}$ Jukka Heikkonen ${ }^{1,3}$ \\ ${ }^{1}$ European Commission, Joint Research Centre, IPSC / Maritime Affairs Unit, Via E. Fermi 2749, 21027 Ispra (VA), Italy \\ ${ }^{2}$ Cefas, Lowestoft Laboratory, Pakefield Road, Lowestoft, Suffolk NR33 0HT, UK \\ ${ }^{3}$ University of Turku, Department of Information Technology, 20014 Turku, Finland
}

\begin{abstract}
Recent studies have drawn attention to the potential for evolutionary changes in lifehistory traits as a consequence of the size-selective process of fishing, and evidence of so-called fisheries-induced evolution has been reported for a number of different species. Most studies of fisheries-induced evolution have focused on changes in sexual maturation using the probabilistic maturation reaction norm method, which requires specific information on the age at which maturation occurs, often derived from macroscopic examination of the gonads. In the absence of sufficiently detailed measurements of maturity it is necessary to derive estimates of the age at which maturation occurs from alternative sources of information, for example, from length at age data. We apply a relatively simple segmented regression model to length at age data for plaice Pleuronectes platessa in the Irish Sea in order to identify the change-point between 2 specific growth schedules that can be used as a proxy for age at first maturity in individual cohorts. We use a Bayesian approach for model fitting and map the resulting distribution of change-points using Gaussian mixture models to show that the age at which the change-point occurred in individual cohorts of both male and female plaice in the Irish Sea has declined progressively over an $18 \mathrm{yr}$ period between 1988 and 2005 .
\end{abstract}

KEY WORDS: Plaice - Maturation · Segmented regression · Reaction norm · Genetic adaptation · Phenotypic plasticity

Resale or republication not permitted without written consent of the publisher

\section{INTRODUCTION}

Growth and reproduction are activities that compete for limited resources. For fish in general, a growth cost of reproduction may be inferred from the frequent observation that the rate of growth decreases with the onset of maturity (Roff 1991, Kozlowski 1996, Stamps et al. 1998). For many fish species, it is typical for initial, juvenile, growth rates to exceed those of older ages and for the trajectory of length with age to follow a non-linear course. Consequently, models used in fisheries research to describe growth generally have smooth, non-linear forms many of which are based on the classical Verhulst logistic growth equation (Tsoularis \& Wallace 2002).
Most commonly the von Bertalanffy growth equation is used, and numerous studies have been conducted to relate physiological processes to von Bertalanffy growth parameters (Beverton 1992, Jensen 1996, Stamps et al. 1998, He \& Stewart 2001, Froese \& Binohlan 2003), most often focussing on the relationship between age at first maturity and asymptotic size.

The term growth cost of reproduction refers to any factor associated with reproduction that reduces the growth rate of a mature individual relative to that of an immature individual of the same length. This cost of reproduction will vary as a function of body size (Charnov 1993, Stamps et al. 1998) and depends on specific activities and physical characteristics associ- 
ated with reproduction, such as (1) competition for resources required for reproduction, e.g. competition for mates or nest sites; (2) the amount of space within the body cavity that can be allocated to reproductive organs; and (3) the costs of locomotion. In all 3 instances, individuals that become reproductive at a larger size might be expected to have a reduced sizespecific cost of reproduction. A life-history strategy of delayed maturation allowing for increased growth might therefore be considered optimal in terms of maximising reproductive potential, but this must be offset against competing processes such as the predation risk of foraging that might favour an alternative strategy of reduced growth and earlier maturation (Mangel \& Stamps 2001). A trade-off therefore exists in the adoption of specific life-history traits that maximise reproductive potential given the competing objectives of increased growth, reduced mortality and reproductive investment. The age at which first maturity occurs is a reflection of the strategic approach that a given species takes to this trade-off and will be highly correlated with other life-history traits, such as growth rate and maximum size.

A number of recent studies have drawn attention to the potential for evolutionary changes in life-history traits as a consequence of the size-selective process of fishing (Rijnsdorp 1993, Law 2000, Heino et al. 2002a), and evidence of so-called fisheries-induced evolution has been reported for a number of different species (Grift et al. 2003, Jørgensen et al. 2007, van Walraven et al. 2010). Most studies of fisheriesinduced evolution have focused on changes in sexual maturation, using the probabilistic maturation reaction norm (PMRN) method developed by Heino et al. (2002b). The PMRN describes the probability of becoming mature at a given size or age. It differs, importantly, from a standard maturity ogive which instead describes the probability of being mature given survival to a specific age or size (Dieckmann \& Heino 2007). Construction of the PMRN from direct observations requires specific information on the age at which maturity first occurs. This information may not always be available. Very often data on maturity indicates whether the individual fish is mature or immature, but does not indicate if it is maturing for the first time. Barot et al. (2004) describe a demographic approach for estimating PMRNs when age at first maturity is unknown. Their method requires a maturity ogive and additional data on growth and mortality and is forced into making a number of simplifying assumptions; for example, that growth rates and mortality remain the same before and after maturation.
In the absence of sufficiently detailed measurements of maturity it is necessary to derive estimates of the age at which maturation occurs from alternative sources of information, for example from length at age data. It has been argued that the theoretical basis for the practical prediction of the age at first maturity from length at age should involve consideration for stage-specific effects of growth (He \& Stewart 2002, Lester et al. 2004, Quince et al. 2008). A potential solution is to apply a 2-stage growth model to length at age data to estimate separate rates of growth either side of a change-point. Segmented regression (Quandt 1958), also called change-point, piece-wise, multi-phase, split-line and 2-phase regression, can be applied to physiological situations where the regression slope is not expected to be constant but to change suddenly at a given point. Such a model may be considered preferable if it provides an improved fit to the length at age; however, its main advantage is that it can be used to identify a point of inflection in the data.

The point of inflection, or change-point, is of particular interest as it may be a marker for some change in physiological response. For example, Lees et al. (1993) applied segmented regression fits to the densities of human female bones and used the changepoint to identify the age at menopause, showing that the age at which it occurred had changed over time. Similarly, the point at which respiration switches from an aerobic to an anaerobic process can be identified from a step change in the ratio of oxygen inhaled versus carbon dioxide exhaled (Julious 2001). Segmented regression has been used to investigate the growth of juvenile rockfish Sebastes jordani (Laidig et al. 1991) where processes such as flexion and juvenile transformation result in different rates of increase in fish length and ototlith radius. It has also been used to describe the trade-off between growth and reproduction in the mussels Mytilus edulis (Salkeld 1995). A variant of segmented regression, often termed the hockey stick or broken stick, has been used to investigate stock and recruitment relationships (Barrowman \& Myers 2000) and as a method of estimating biomass reference points for management under the precautionary approach (O'Brien \& Maxwell 2002). With specific reference to estimating the onset of maturity, Rijnsdorp \& Storbeck (1995) applied the segmented regression approach to back-calculated growth data from otolith measurements of North Sea plaice Pleuronectes platessa, and, more recently, Baulier \& Heino (2008) investigated its application to growth patterns in Atlantic herring Clupea harengus, although neither 
study found the approach to be entirely satisfactory, recommending instead that a bio-energetic modelling approach may provide better estimates. Mollet et al. (2010) extended the method to estimate multiple life-history parameters using the segmented regression approach to fit an energy allocation model to individual growth trajectories for female North Sea plaice. Their method provided estimates of the age of the onset of maturation that corresponded well with those obtained from direct observation.

In the present study, we investigate the potential for a simple, mechanistic model to be used as a basic indicator of long-term trends in maturation schedules. Our approach is relatively simple and conceptually similar to that of Rijnsdorp \& Storbeck (1995), but instead of back-calculated estimates of length at age from individual fish we use direct observations of length at age, at the population level, obtained from a research survey. Further, by considering a longer time series of data, we are able to investigate potential trends in maturation schedules. We apply a simple segmented regression model to length at age data, for plaice in the Irish Sea, to identify the change-point between 2 specific growth schedules that may be used as a proxy for age at first maturity in individual cohorts. We compare the estimates obtained from the segmented regression approach with those derived from the PMRN method. For model fitting and uncertainty analysis we use a Bayesian approach based on the Markov Chain Monte Carlo (MCMC) method. Plaice exhibit strong sexual dimorphism, and males and females show differences in both the onset of sexual maturation and the extent of resource allocation to reproduction (Rijnsdorp \& Ibelings 1989). The study is therefore conducted on a separate sex basis.

\section{METHODS}

Numerous parameterisations of the segmented regression model exist. For a simple 2-line case the model may be expressed as:

$$
y_{i}=\left\{\begin{array}{lll}
\beta_{0}+\beta_{1} x_{i}+\varepsilon_{i} & \text { for } & x_{i} \leq \delta \\
\beta_{0}+\beta_{1} x_{i}+\beta_{2}\left(x_{i}-\delta\right)+\varepsilon_{i} & \text { for } & x_{i}>\delta
\end{array}\right.
$$

where $y_{i}$ is the value of the $i$ th observation (length, $\mathrm{mm}$ ) and $x_{i}$ is the corresponding value for the independent variable (age, yr), $\delta$ is the location of the change-point on the $x$-axis and $\varepsilon_{i}$ are assumed to be independent and identically distributed. The intercept is $\beta_{0}$ and the slopes of the 2 lines are $\beta_{1}$ and $\beta_{1}+$ $\beta_{2}$, respectively. Parameterising the model in this way forces continuity at the change-point $(\delta)$. The function is therefore continuous, though not differentiable, at the change-point. When the location of the change-point is unknown, the problem cannot be solved simply using linear algebra. Consequently, the fitting of the segmented regression model occurs in 2 stages using a method similar to that described by Julious (2001).

In the above 2-line model, the position of the change-point must first be determined before the 2 limbs of the regression can be estimated. An alternative parameterisation yields a continuous function that gives a smooth transition through the changepoint (Seber \& Wild 2003) such that:

$$
y_{i}=\theta_{0}+\theta_{1}\left(x_{i}-\delta\right)+\theta_{2} \sqrt{\left(x_{i}-\delta\right)^{2}+\gamma}+\varepsilon_{i}
$$

where $\gamma$ controls the extent of curvature at the change-point ( $\delta$ ) such that as $\gamma$ tends toward zero the function approaches the simple 2-line model above. This function is both continuous through the changepoint and differentiable enabling the model to be fitted in a single process. The slopes of the 2 limbs of the curve can be controlled by applying constraints to the parameter estimates. A negative slope for the second limb can be prevented by forcing $\theta_{1}$ to be positive and $\theta_{2}$ to be negative with an absolute value $\leq \theta_{1}$. Under these conditions, the slope of the first limb is given by $\theta_{1}-\theta_{2}$, and the slope of the second limb, by $\theta_{1}+\theta_{2}$. It follows that when $\theta_{2}$ is constrained to be negative and to have an absolute value equal to $\theta_{1}$, the slope of the second limb becomes zero, forming the so-called hockey stick, or broken stick model. Since a decline in length with age was considered to be biologically unrealistic, the model was constrained to have a non-negative slope in the second limb. All subsequent analyses are based on this continuous segmented regression model.

The data used in this exercise were separate sex length at age observations for plaice Pleuronectes platessa in the Irish Sea, obtained from a third quarter research survey for the period 1989 to 2009. Previous studies have found significant spatial variation in the dynamics of reproduction and growth for plaice in the Irish Sea (Horwood 1990, Nash et al. 2000). Consequently, samples were only considered from the northeastern region of the Irish Sea, comprising Liverpool Bay, Morecambe Bay and the area to the east and south of the Isle of Man. Before fitting, all ages were incremented by 0.75 to reflect the period of the year in which the survey took place. Although the data available represented cohorts from 1970 through 2009, many of these cohorts contained only partial age ranges and only the 1988 to 
2005 cohorts were considered to contain sufficient information to enable meaningful estimation of the change-point.

The model was fitted to individual cohorts on a separate sex basis using a Bayesian approach. The key principle of the Bayesian approach is to construct the posterior probability distribution for all unknown entities in a model given the data (Gelman et al. 2003). Use of the posterior probabilities requires explicit definition of the prior probabilities for the parameters and the formulation of the likelihood function of the model based on the residual model. Bayesian analysis has many benefits over the traditional maximum likelihood approach, one of which is the model uncertainty analysis based on its posterior probability distribution (see e.g. Gilks et al. 1996, Gelman et al. 2003). Because for almost all cases direct analytical integrations of the posterior probability distributions are impossible to perform, numerical methods such as MCMC approaches that attempt to draw samples from the distributions of interest are commonly used (Gilks et al. 1996). One particular MCMC method, the Gibbs sampler (Geman \& Geman 1984), has been very widely applied to a broad class of Bayesian problems.

We used OpenBugs software (Lunn et al. 2009), which is designed specifically for the Bayesian analysis of statistical models using MCMC methods. Since our primary interest was in change-point determination, we used non-informative priors for the model parameters allowing the information from the data to determine the posterior distribution of the estimated change-point. For the model parameters that were constrained to a positive value we used flat gamma priors. For the other parameters, priors were defined as uniform distributions. To accommodate the constraints in the smooth transition function (i.e. the requirement $\left|\theta_{2}\right| \leq \theta_{1}$, where $\theta_{2} \leq 0$ ) nested gamma priors were defined for these parameters. The model residuals were assumed to be normally distributed. In order to determine how many steps in MCMC sampling were needed to converge to the stationary posterior distributions within an acceptable error, we used potential scale reduction factor (PSRF) statistics (Brooks \& Gelman 1998). Finally, for change-point posterior distribution estimation and visualization, Gaussian mixture models (GMMs) (Bishop 2006) were fitted to the resulting change-point samples.

We calculated the PMRN for Irish Sea plaice following the approach of Mollet et al. (2007). This approach incorporated the method of Barot et al. (2004) for the estimation of first time spawners and assumed an underlying von Bertalanffy growth model. Maturity observations, taken from the same samples for which age and length were obtained and determined from macroscopic inspection, were used to estimate a maturity ogive using a generalised linear model (GLM) with binomial response and the explanatory variables age, length and period. As for the segmented regression approach, the data were aggregated by cohort into 3 periods, each comprising 6 cohorts with individual growth models and maturity ogives fitted for each period and sex.

The simplistic nature of the segmented regression model is appealing and provides the possibility to investigate more than just the position of the changepoint. Provided the fitted model does not deviate markedly from the observed length at age at the oldest or youngest ages, it is possible to make a very simple, although somewhat crude, comparison of growth rates before and after maturation. The relationship between the slopes of the 2 limbs was compared using a simple linear regression to investigate to what extent pre-maturity growth rate might be related to post-maturity growth rate and whether this relationship differs between the sexes.

\section{RESULTS}

Mean length at age and the corresponding fitted growth model for individual cohorts of male and female plaice Pleuronectes platessa are shown in Figs. $1 \& 2$, and parameter estimates for each of the fitted models are given in Table 1. Median estimates of the change-point and corresponding probability intervals derived from MCMC sampling (Figs. 3 \& 4) for individual cohorts of female and male plaice, respectively, show that for both sexes there has been a general decline in the age at which the changepoint occurs. This would indicate a reduction in the age at first maturity and the point at which growth switches from the juvenile to the post-maturity schedule. Linear regressions of the median changepoint estimates against cohort gave significant negative slopes of 0.07 cohort $^{-1}$ for females and 0.06 cohort $^{-1}$ for males, which would suggest an overall reduction in the age at first maturity of $1.3 \mathrm{yr}$ in females and $1.1 \mathrm{yr}$ in males over the $18 \mathrm{yr}$ period from 1988 to 2005. The direction of this shift is consistent with studies of plaice in the North Sea which, using the PMRN approach, have also detected a significant reduction in the age at first maturity (van Walraven et al. 2010). However, the scale of the reduction detected here is greater than that found in other studies. 


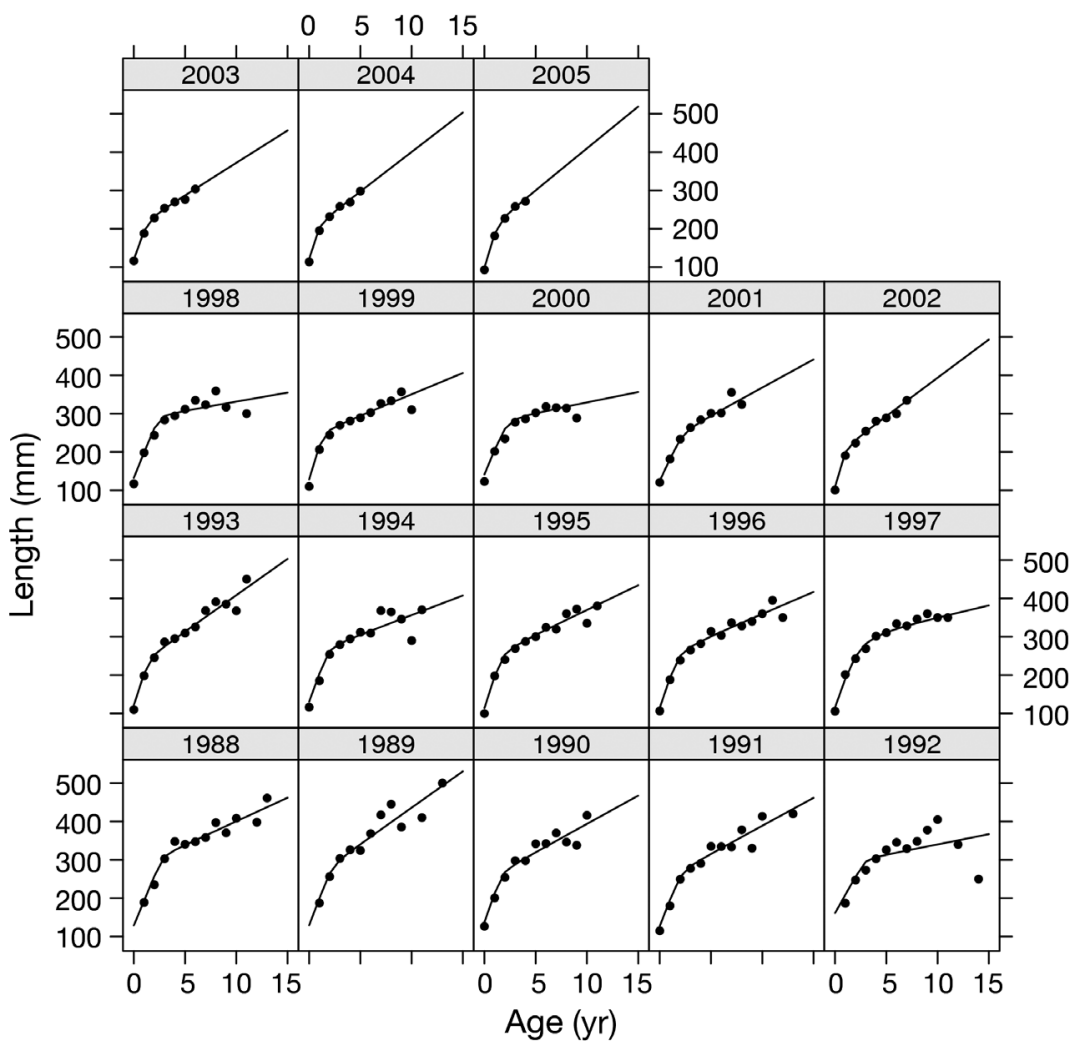

Fig. 1. Pleuronectes platessa. Mean length at age and fitted segmented regression growth model for individual cohorts (1988 to 2005) of female plaice in the Irish Sea
Until 1982, separate sex estimates of the proportion mature at age for Irish Sea plaice were used for assessment purposes (ICES, 2009). These proportions indicate very approximate age at $50 \%$ maturity $\left(\mathrm{A} 50_{\mathrm{M}}\right)$ values of around $2.5 \mathrm{yr}$ for males and 3.0 to $3.5 \mathrm{yr}$ for females. The results of the segmented regression approach (conducted here for a slightly more recent period) indicate that males and females have more similar ages at first maturity with average predicted values for the 1988 to 1993 cohorts of approximately 2.7 for males and 2.9 for females. The results obtained here are therefore comparable to previous maturity estimates for Irish Sea plaice.

Posterior probability distributions (aggregated over 6 yr periods) of the change-point estimates (Figs. 5 \& 6) show a marked decline, in both sexes, between the first (1988 to
1993) and second (1994 to 1999) periods in both the age and length at which first maturity occurs. A much smaller change is detected for the third period (2000 to 2005). The $95 \%$ probability contours are wider for females than for males, reflecting greater uncertainty in the true location of the change-point. The posterior probability distributions indicate a slowing of the trend towards earlier maturation at smaller sizes, although no clear trajectory can be identified from the data available.

The results of the PMRN analysis (Fig. 7) also show a progressive reduction in Lp50 (the length at which the probability of becoming mature is $50 \%$ ) over the 3 periods for females, whilst, for males, the Lp50 contour declined markedly between the first and second period but remained relatively stationary thereafter. Interpolation of the growth models and the PMRN yielded approximate point estimates of $2.9,257 ; 2.6,248$; and

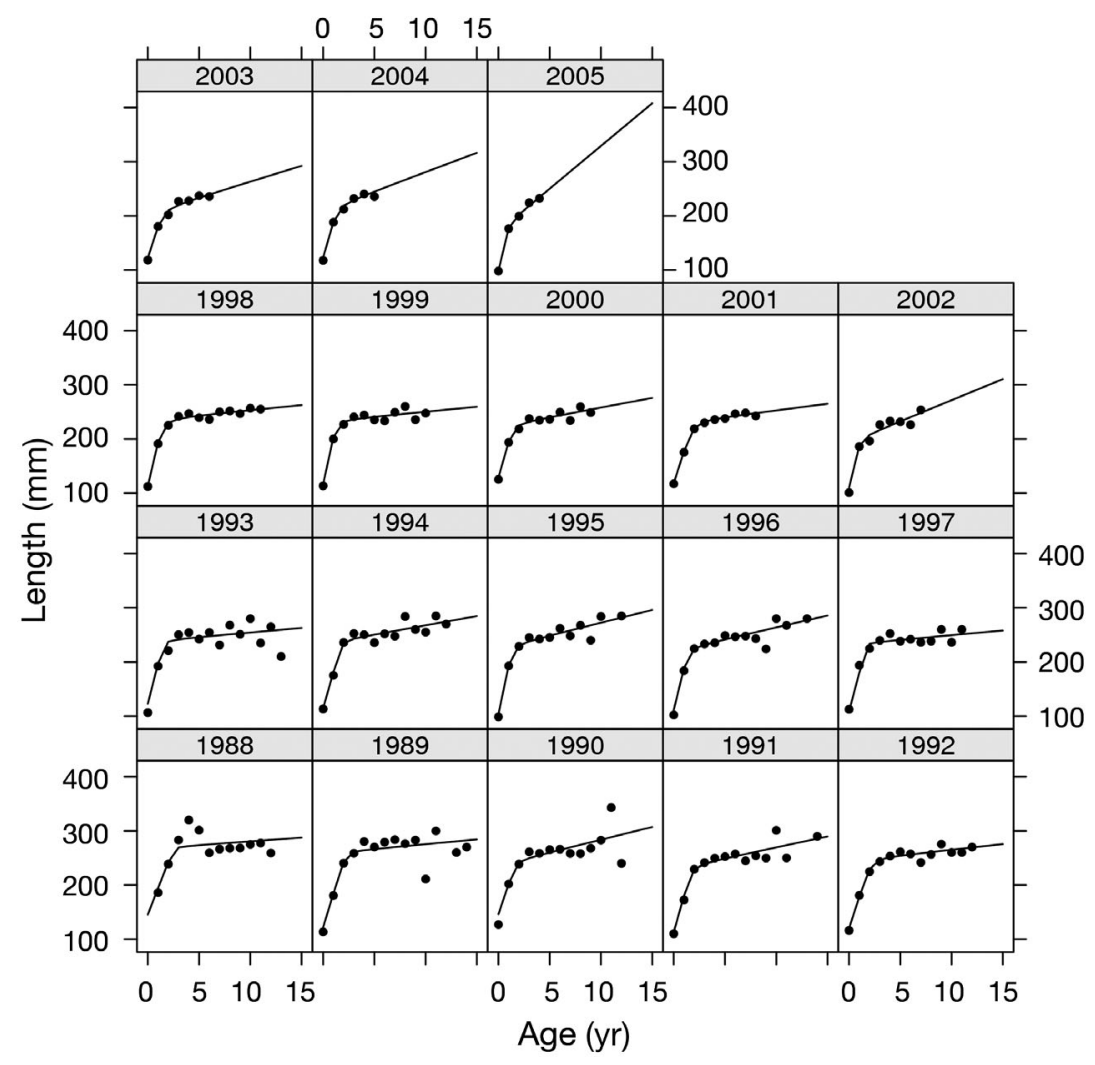

Fig. 2. Pleuronectes platessa. As in Fig. 1, but for males 
Table 1. Pleuronectes platessa. Segmented regression parameter estimates for individual cohorts (1988 to 2005) of male and female plaice from the Irish Sea

\begin{tabular}{|c|c|c|c|c|c|c|c|c|c|c|}
\hline \multirow[t]{2}{*}{ Cohort } & \multicolumn{5}{|c|}{-Females } & \multirow[b]{2}{*}{ Delta } & \multirow[b]{2}{*}{ Gamma } & _Males- & \multirow[b]{2}{*}{ Theta_1 } & \multirow[b]{2}{*}{ Theta_2 } \\
\hline & Delta & Gamma & Theta_0 & Theta_1 & Theta_2 & & & Theta_0 & & \\
\hline 1988 & 3.57 & 0.15 & 312.3 & 38.6 & 26.3 & 3.29 & 0.08 & 270.5 & 25.3 & 23.9 \\
\hline 1989 & 2.96 & 0.29 & 289.3 & 45.3 & 26.4 & 2.97 & 0.12 & 262.1 & 32.2 & 30.5 \\
\hline 1990 & 2.44 & 0.23 & 272.0 & 46.4 & 31.7 & 2.44 & 0.16 & 245.0 & 31.3 & 26.6 \\
\hline 1991 & 2.81 & 0.34 & 274.2 & 42.4 & 27.9 & 2.51 & 0.20 & 236.7 & 36.7 & 32.6 \\
\hline 1992 & 3.69 & 0.20 & 303.1 & 26.7 & 21.4 & 2.88 & 0.29 & 249.4 & 31.0 & 28.9 \\
\hline 1993 & 2.19 & 0.17 & 246.5 & 52.5 & 33.5 & 2.27 & 0.10 & 240.3 & 39.2 & 37.5 \\
\hline 1994 & 2.63 & 0.23 & 274.1 & 42.7 & 32.5 & 2.60 & 0.09 & 240.0 & 35.5 & 32.1 \\
\hline 1995 & 2.35 & 0.45 & 263.4 & 51.4 & 38.6 & 2.05 & 0.17 & 232.0 & 50.6 & 45.9 \\
\hline 1996 & 2.48 & 0.55 & 266.2 & 47.9 & 36.5 & 2.13 & 0.17 & 226.5 & 43.1 & 38.8 \\
\hline 1997 & 2.72 & 2.16 & 306.5 & 46.5 & 40.5 & 2.50 & 0.03 & 235.2 & 36.9 & 35.2 \\
\hline 1998 & 3.15 & 0.25 & 296.3 & 36.1 & 31.5 & 2.05 & 0.37 & 238.4 & 46.9 & 45.1 \\
\hline 1999 & 2.06 & 0.21 & 254.6 & 52.5 & 41.5 & 1.96 & 0.18 & 235.0 & 48.7 & 46.9 \\
\hline 2000 & 2.89 & 0.53 & 288.1 & 36.0 & 30.7 & 2.11 & 0.22 & 227.9 & 37.4 & 33.9 \\
\hline 2001 & 2.91 & 0.44 & 254.0 & 36.7 & 22.1 & 2.55 & 0.43 & 233.8 & 32.4 & 30.0 \\
\hline 2002 & 1.65 & 0.21 & 212.9 & 65.0 & 45.1 & 1.78 & 0.12 & 202.3 & 47.6 & 39.9 \\
\hline 2003 & 1.95 & 0.34 & 224.8 & 50.4 & 33.6 & 2.09 & 0.40 & 213.6 & 35.3 & 29.5 \\
\hline 2004 & 1.70 & 0.16 & 214.8 & 57.8 & 37.3 & 2.02 & 0.32 & 219.7 & 40.1 & 33.0 \\
\hline 2005 & 1.96 & 0.23 & 218.8 & 59.3 & 37.5 & 1.78 & 0.07 & 188.0 & 50.7 & 34.9 \\
\hline
\end{tabular}

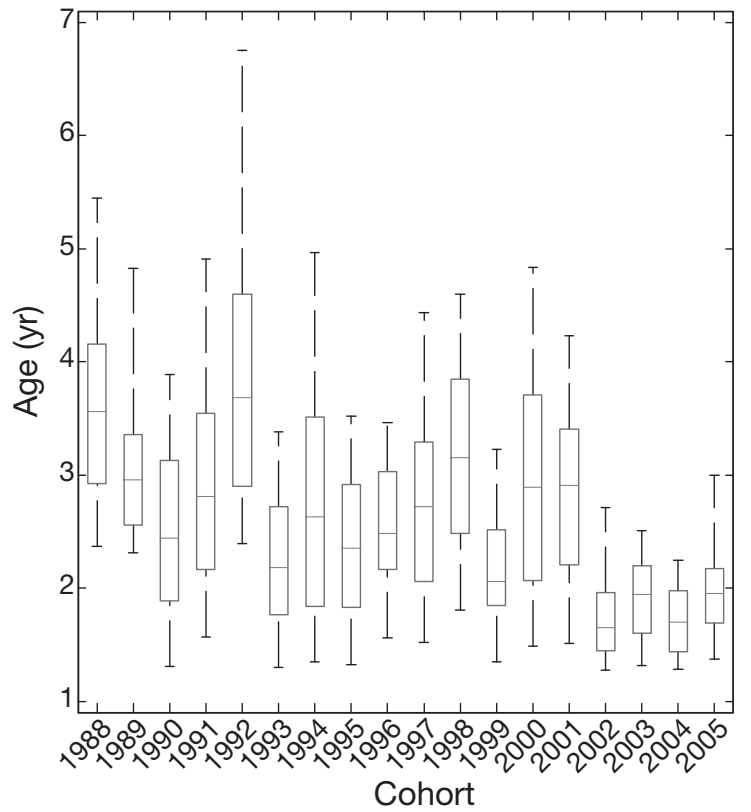

Fig. 3. Pleuronectes platessa. Estimates of the age (yr, females) at which the segmented regression change-point occurs based on Bayesian modelling using Markov Chain Monte Carlo (MCMC) procedure. Boxes show median, 25th and 75th percentiles; whiskers show 5th and 95th percentiles of the MCMC samples for the change-point variable

2.2, 216 for the age (yr) and length (mm), respectively, of first maturity for females in each period, and of 2.5, 221; 1.9, 194; and 2.2, 198 for males. For females these point estimates compare relatively well with the estimates derived from the segmented

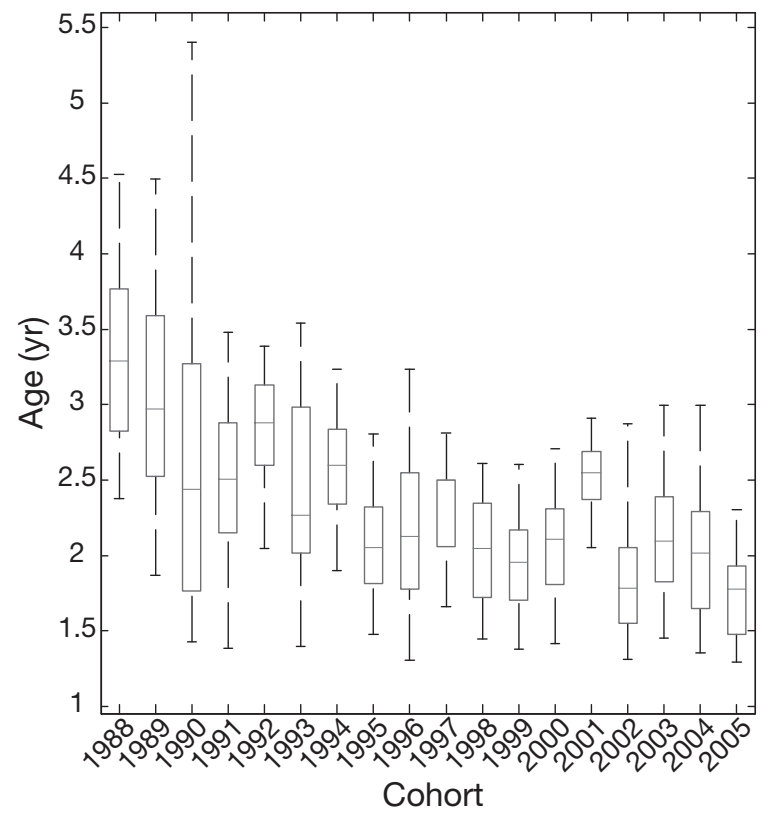

Fig. 4. Pleuronectes platessa. As in Fig. 3, but for males

regression, each point lying inside the $95 \%$ contour of the posterior probability distribution of the segmented regression change point. For males however, the point estimates show a similar trend but lie consistently outside the lower left limits of the posterior probability distributions.

The ability to effectively determine the position of the change-point can depend on the number of points either side of it. As the change-point moves 


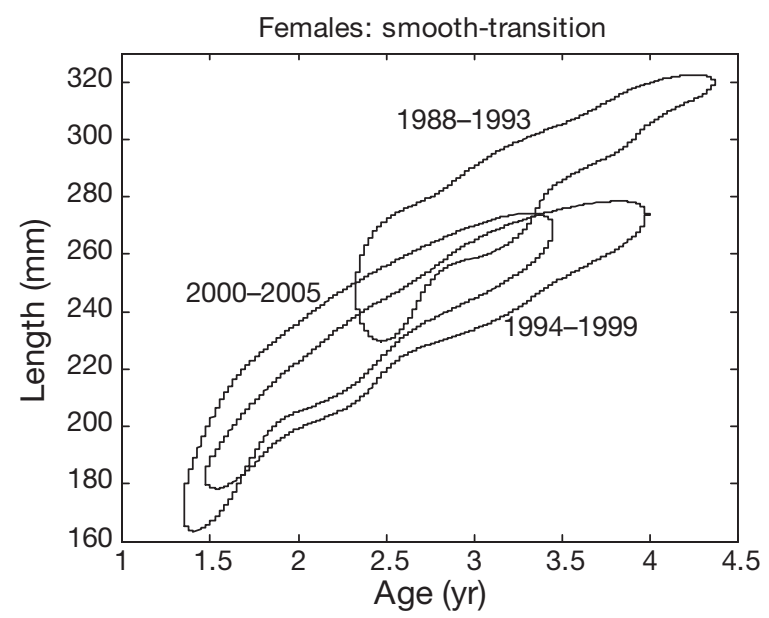

Fig. 5. Pleuronectes platessa. Posterior probability distribution of change points (aggregated over 6 yr periods) based on Markov Chain Monte Carlo sampling for female plaice; the change point falls inside the contour lines with $95 \%$ probability

closer to the upper or lower limit of the data, it will become more difficult to detect. For example, the 1988 cohort has fewer observations at younger ages and the change-point might be expected to be close to the lower limit of the age range of available data. This may result in over-estimation of the true change-point level. Conversely, the 2005 cohort has fewer observations of older fish. The change-point occurs closer to the upper limit of the age range of available data, and this may lead to under-estimation of the true level at which the change-point occurs. It is possible, therefore, that a perceived decline in the age at which the change-point occurs could merely be an artefact of the range of ages available in each cohort. To investigate the extent of this potential bias, change-point estimates were derived for each cohort using only the age range of 0 to $5 \mathrm{yr}$ and also using the full range of available data. The results (Fig. 8) do indeed indicate a tendency to estimate a lower change-point level in some cohorts when older ages are not represented in the data and the very low estimates for the 2003, 2004 and 2005 cohorts may be a consequence of this. But the direction and magnitude of the overall downward trend remained unaffected when the age range of the analysis was reduced.

Estimation of the change-point is also affected by the difference between the slopes of the 2 limbs of the fitted model. Simulation exercises, conducted for the simple 2-line model, have shown that the power of the test increases as the difference between the 2 regression slopes increases (Julious 2001). Female plaice have a higher post-maturity growth rate than

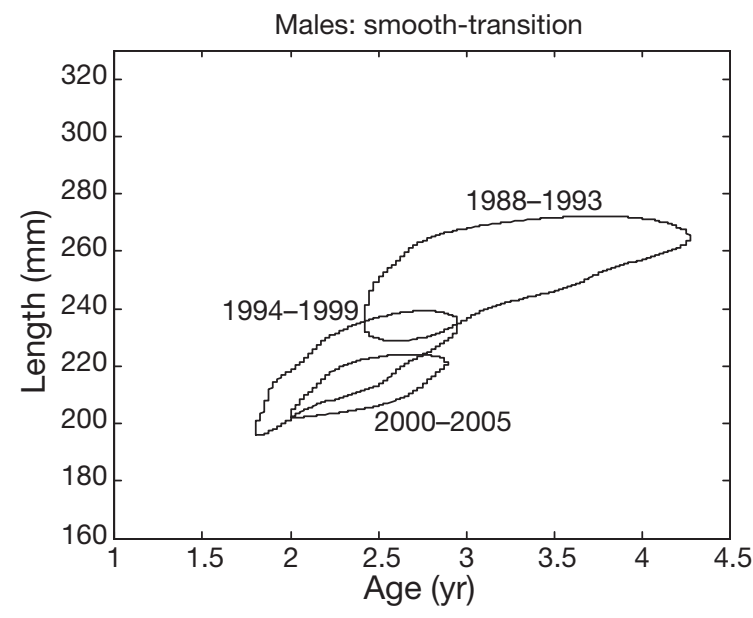

Fig. 6. Pleuronectes platessa. As in Fig. 5, but for males; the change point falls inside the contour lines with $95 \%$ probability

males, and the difference in gradient between the 2 slopes is therefore smaller in females. As a consequence, probability intervals around the changepoint estimates are generally greater for females than for males. For males, the change in growth rate between the pre-maturity and post-maturity phases

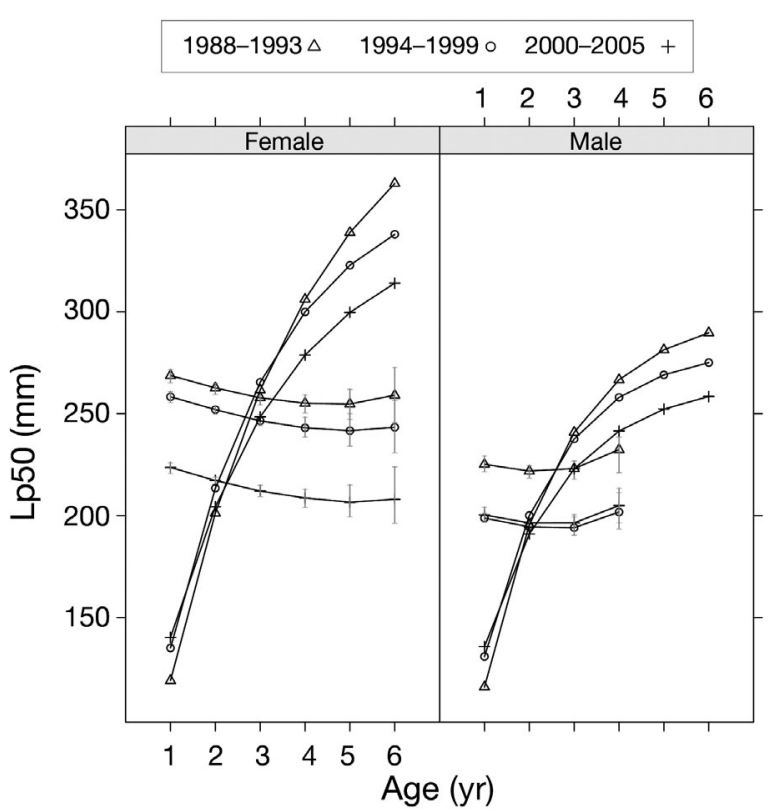

Fig. 7. Pleuronectes platessa. Probabilistic maturation reaction norms (PMRNs) for female and male plaice in the Irish Sea for three 6 yr periods from 1988 to 2005. Lp50: length at which probability of becoming mature is $50 \%$. Error bars on PMRN lines show the 5th and 95th percentiles calculated from 100 samples from a non-parametric bootstrap. The von Bertalanffy growth curves, fitted to each of the 3 periods, are also shown 


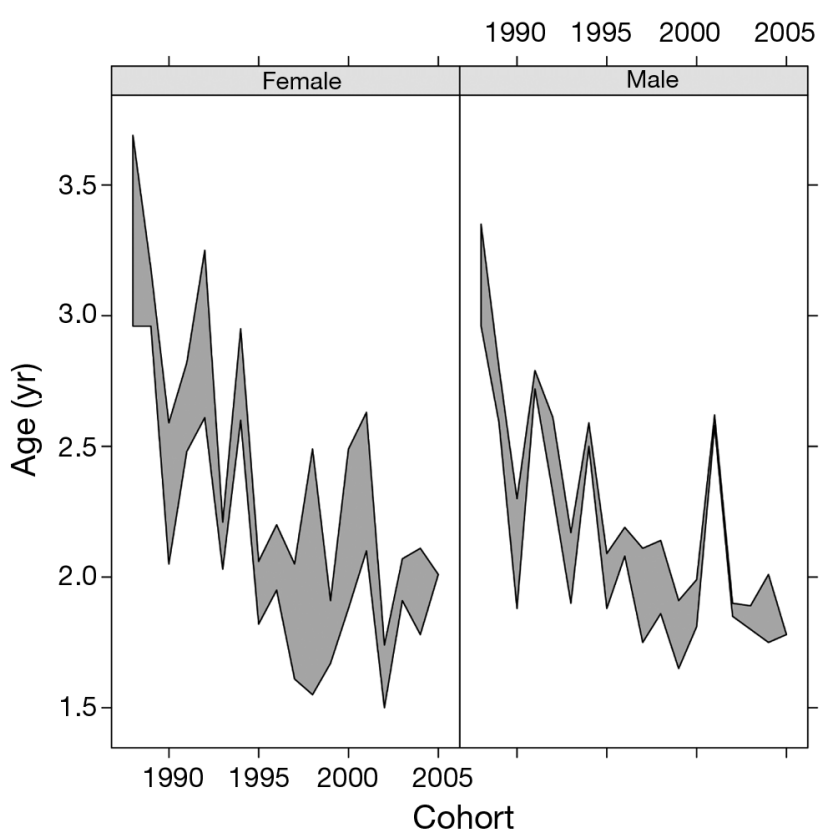

Fig. 8. Pleuronectes platessa. Model sensitivity analysis. Shaded region shows the upper and lower bounds of the medians of the Markov Chain Monte Carlo change-point estimates for models fitted to a restricted age range ( 0 to $5 \mathrm{yr}$ ) and to all of the available data for the 2-line model and the smooth transition model

was particularly abrupt, with growth rates declining dramatically following maturation.

For females, a significant positive correlation was found between pre-maturity and post-maturity growth rates (Fig. 9). Cohorts with fast-growing juveniles were found to also have a faster growth rate as adults, whereas cohorts that grew more slowly as juveniles also had slow post-maturity growth rates. No significant relationship was found for males where post-maturity growth rates appeared to vary independently of the pre-maturity rate.

\section{DISCUSSION}

The progressive reduction in the age at which the change-point occurs in both male and female plaice Pleuronectes platessa is striking and implies that the age at first maturity in both sexes has declined over time. The results of the sensitivity analysis in which models were fitted to a truncated age range show that this progressive reduction in age at maturity is not merely an artefact of the number of ages to which the model was fitted. However, the range of cohorts available for the present study was relatively short and it is difficult to obtain a clearer picture without analysing a longer time series. Commercial catch data exist for a longer time period, but were not considered appropriate for our study as the minimum landing size $(27 \mathrm{~cm})$ imposes a strong bias on the length distribution of fish observed on the market. The vast majority of male plaice and a large proportion of female plaice appearing on the market will be fully mature.

The length at age data used in the present study were obtained from a third quarter research survey. Prior to analysis, all ages were incremented by 0.75 so that the change-point estimates corresponded appropriately to the beginning of the year in which the measurements were made. However, as Nash \& Geffen (2005) point out, recruit spawners have to build up energy reserves but do not have to recover from depleted body condition from the previous year's spawning. Reproduction will thus have its maximum effect on somatic growth after the first reproductive season. The point at which energy resources become limiting for growth and the time at which this manifests itself as a reduction in growth rate may therefore occur sometime after maturation. Consequently, the change-point estimates presented here may over-estimate age at first maturity.

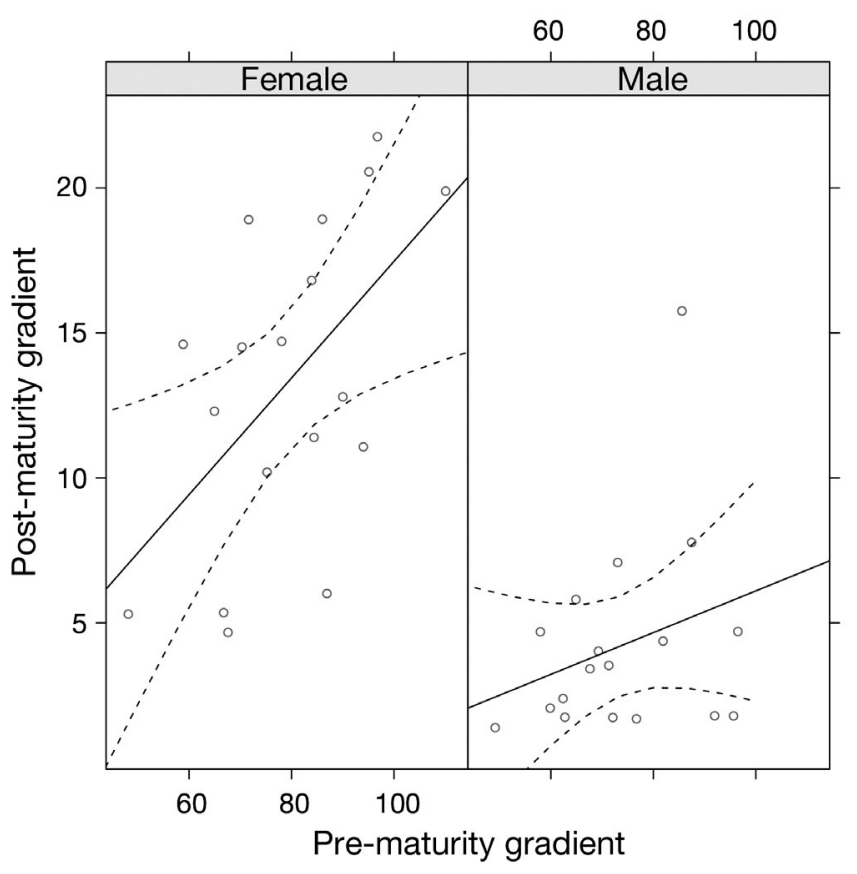

Fig. 9. Pleuronectes platessa. Linear regression showing the relationships between the gradients of the pre-maturity $\left(\theta_{1}+\theta_{2}\right)$ and post-maturity $\left(\theta_{1}-\theta_{2}\right)$ growth schedules for individual cohorts (1988 to 2005) of male and female plaice from the Irish Sea (females, slope $=0.201$, p-value $=0.016$; males, slope $=0.072$, p-value $=0.254$ ). Dotted lines show $95 \%$ confidence intervals about the fitted model 
PMRMs extend the theory of the deterministic reaction norm to allow a probabilistic description of the maturation process. The PMRN approach has been applied in a number of cases (see Dieckmann \& Heino 2007) to identify long-term trends in maturation schedules for a variety of different species. Direct calculation of the PMRN requires representative data describing the age and size of both newly matured and immature individuals. In many instances, these data are missing, either due to spatial separation of the mature and immature stock components or else because it is not possible to distinguish between first-time and repeat spawning fish. The demographic method of Barot et al. (2004) reconstructs the necessary data that are required to compute the PMRN using snapshots of data from 2 or more separate sampling periods. The method relies on a number of simplifying assumptions, most notably that growth rates do not change substantially between the pre- and post-maturity phases. Barot et al. (2004) tested the performance of their method to violations of this basic assumption and concluded that, with a sufficiently large sample size, the method was robust to a $25 \%$ difference between pre- and post-maturity growth rates.

A comparison of the direct and demographic methods (Pérez-Rodríguez et al. 2009) found good correspondence between the 2 approaches in spite of potential violation of the assumptions underpinning the demographic method. Their results showed that the estimates from the 2 methods were highly correlated, and they concluded that the demographic method was a reliable way of calculating PMRNs for situations where data are insufficient to separate recruit and repeat spawners. Interestingly, they found a small but consistent bias between the 2 methods with the demographic method producing estimates of Lp50 that were on average $2.4 \mathrm{~cm}$ lower than those determined from the direct method. We also found the estimates derived from the demographic method to be slightly lower than the estimates derived from the segmented regression approach. Unfortunately, without the necessary data to conduct a comparison using the direct method, we were unable to draw any firm conclusions, and we can only speculate on the possible causes of this bias.

Perhaps the most likely cause for this bias is the performance of the assumed growth model when reconstructing the data necessary to calculate the PMRN. We found the discrepancy between the segmented regression estimates of Lp50 and those of the PMRN to be greater for males than for females. Males exhibit a much greater reduction in somatic growth rate following maturation and a sharper transition between the apparent pre- and post-maturity growth schedules. During initial analyses we found the fits of the von Bertalanffy growth model were almost always poorer for males than for females. Lester et al. (2004) found the von Bertalanffy equation to be an appropriate model for post-maturity somatic growth but not appropriate for pre-maturation growth, and it is argued that multi-phase models may provide a more appropriate representation of growth in fishes (Dumas et al. 2007, Quince et al. 2008). These findings support the further investigation of alternative growth assumptions when investigating trends in maturation schedules, and the approach of Mollet et al. (2010) has already begun this process through the incorporation of a bio-energetic approach to energy allocation.

Bio-energetic considerations alone, however, may not be sufficient to fully explain the differences between the sexes or the trends observed over time. The sexual dimorphism in growth cannot be fully explained by differences between the sexes in reproductive investment and the onset of sexual maturity (Rijnsdorp \& Ibelings 1989). The possibility of behavioural differences between the sexes during spawning, such as swimming activity (Solmundsson et al. 2003), male-male competition and size-assortive mating preferences, has also been suggested as a potential factor for the determination of life-history traits in plaice (Rijnsdorp et al. 2010).

It should be stressed that the differences detected in the present study between the 2 methods for estimating trends in maturation schedules represent a relatively small but consistent bias and that the overall direction and magnitude of the reductions in Lp50 were found to be very similar through the 2 approaches. Although it would be relatively straightforward to substitute an alternative growth model into the PMRN calculations, we have not done this here. Our intention was merely to illustrate the potential for the change-point, as estimated from a simple segmented regression model, to be used as a basic indicator of long-term trends in the age and length at first maturity. Neither do we attempt to explain why such changes in maturation might occur. In this respect, we refer the reader to the more theoretical approaches of Dunlop et al. (2009) and Poos et al. (2011), which also both allow for stage-specific growth.

The estimation of age and length at maturity using segmented regression to detect a change point in the growth rate of a cohort is a simple, mechanistic technique that requires only length at age data. The approach enables estimation of the age, and length, 
at which maturation occurs to be derived when only length at age data are available, and it enables the investigation of long-term trends in the age at which first maturation occurs at a population level. However, as previous studies have shown, the method is not without its limitations. The approach does require a sufficient time series of cohort-based measurements of length at age that cover a sufficient age range to enable meaningful estimation of the change-point. In this respect, the approach may not be appropriate for very short-lived species, such as small pelagics, or for very heavily exploited stocks that have severely truncated age ranges.

Acknowledgements. The authors are very grateful to Adriaan Rijnsdorp and 2 other reviewers for helpful comments and suggestions on an earlier draft of this paper and to colleagues from Cefas and the FISHREG action at the European Commission's Joint Research Centre for helpful discussions and suggestions. In particular we are very grateful to Fabian Mollet for the provision of $\mathrm{R}$ code and helpful advice for calculating the PMRN.

\section{LITERATURE CITED}

Barot S, Heino M, O'Brien L, Dieckmann U (2004) Estimating reaction norms for age and size at maturation when age at first maturation is unknown. Evol Ecol Res 6: 659-678

Barrowman NJ, Myers RA (2000) Still more spawnerrecruitment curves: the hockey stick and its generalisations. Can J Fish Aquat Sci 57:665-676

Baulier L, Heino M (2008) Norwegian spring-spawning herring as the test case of piecewise linear regression method for detecting maturation from growth patterns. J Fish Biol 73:2452-2467

> Beverton RJH (1992) Patterns of reproductive strategy parameters in some marine teleost fishes. J Fish Biol 41: 137-160

Bishop CM (2006) Pattern recognition and machine learning, Springer, Heidelberg

> Brooks SP, Gelman A (1998) General methods for monitoring convergence of iterative simulations. J Comput Graph Statist 7:434-455

Charnov EL (1993) Life history invariants. Some explorations of symmetry in evolutionary ecology. Oxford University Press, Oxford

> Dieckmann U, Heino M (2007) Probabilistic maturation reaction norms: their history, strengths, and limitations. Mar Ecol Prog Ser 335:253-269

> Dumas A, France J, Bureau DP (2007) Evidence of three growth stanzas in rainbow trout (Oncorhynchus mykiss) across life stages and adaptation of the thermal-unit growth coefficient. Aquaculture 267:139-146

Dunlop ES, Heino M, Dieckmann U (2009) Eco-genetic modeling of contemporary life-history evolution. Ecol Appl 19:1815-1834

Froese R, Binohlan C (2003) Simple methods to obtain preliminary growth estimates for fishes. J Appl Ichthyol 19: 376-379

Gelman A, Carlin JB, Stern HS, Rubin DB (2003) Bayesian data analysis, 2nd edn. CRC Press, Boca Raton, FL

Geman S, Geman D (1984) Stochastic relaxation, Gibbs distributions and the Bayesian restoration of images. IEEE Trans Pattern Anal Mach Intell 6:721-741

Gilks W, Richardson S, Spiegelhalter D (1996) Markov Chain Monte Carlo in Practice. Chapman \& Hall, New York, NY

Grift RE, Rijnsdorp AD, Barot S, Heino M, Dieckmann U (2003) Fisheries-induced trends in reaction norms for maturation in North Sea plaice. Mar Ecol Prog Ser 257: 247-257

He JX, Stewart DJ (2001) Age and size at first reproduction of fishes: predictive models based only on growth trajectories. Ecology 82:784-791

$>$ He JX, Stewart DJ (2002) A stage-explicit expression of the von Bertalanffy growth model for understanding age at first reproduction of Great Lakes fishes. Can J Fish Aquat Sci 59:250-261

Heino M, Dieckmann U, Godø R (2002a) Reaction norm analysis of fisheries induced adaptive change and the case of the Northeast Arctic cod. ICES CM 2002/Y:14

> Heino M, Dieckmann U, Godø R (2002b) Estimating reaction norms for age and size at maturation with reconstructed immature size distributions: a new technique illustrated by application to Northeast Arctic cod. ICES J Mar Sci 59:562-575

$>$ Horwood JW (1990) Fecundity and maturity of plaice (Pleuronectes platessa) from Cardigan Bay. J Mar Biol Assoc UK 70:515-529

ICES (International Council for Exploration of the Sea) (2009) Stock annexes to the report of the Working Group on the Celtic Seas Ecoregion (WGCSE). Stock annexes to ICES CM 2009/ACOM:09

Jensen AL (1996) Beverton and Holt life history invariants result from optimal trade-off of reproduction and survival. Can J Fish Aquat Sci 53:820-822

Jørgensen C, Enberg K, Dunlop ES, Arlinghaus R and others (2007) Managing evolving fish stocks. Science 318: $1247-1248$

> Julious SA (2001) Inference and estimation in a changepoint regression problem. Statistician 50:51-61

> Kozlowski J (1996) Optimal allocation of resources explains inter-specific life history patterns in animals with indeterminate growth. Proc Biol Sci 263:559-566

Laidig TE, Ralston S, Bence JR (1991) Dynamics of growth in the early life history of shortbelly rockfish (Sebastes jordani). Fish Bull 89:611-621

> Law R (2000) Fishing, selection and phenotypic evolution. ICES J Mar Sci 57:659-668

> Lees B, Molleson T, Arnett TR, Stevenson JC (1993) Differences in proximal femur bone density over two centuries. Lancet 341:673-675

> Lester NP, Shuter BJ, Abrams PA (2004) Interpreting the von Bertalanffy model of somatic growth in fishes: the cost of reproduction. Proc Biol Sci 271:1625-1631

Lunn D, Spiegelhalter D, Thomas A, Best N (2009) The BUGS project: evolution, critique and future directions (with discussion). Stat Med 28:3049-3067

Mangel M, Stamps J (2001) Trade-offs between growth and mortality and the maintenance of individual variation in growth. Evol Ecol Res 3:583-593

Mollet F, Kraak SBM, Rijnsdorp AD (2007) Fisheriesinduced evolutionary changes in maturation reaction norms in North Sea sole Solea solea. Mar Ecol Prog Ser 351:189-199 
Mollet F, Ernande B, Brunel T, Rijnsdorp AD (2010) Multiple growth-correlated life history traits estimated simultaneously in individuals. Oikos 119:10-26

Nash RDM, Geffen AJ (2005) Age and growth. In: Gibson RN (ed) Flatfishes, biology and exploitation. Blackwell Science, Oxford

Nash RDM, Whitthames PR, Pawson M, Alesworth E (2000) Regional variability in the dynamics of reproduction and growth of Irish Sea plaice, Pleuronectes platessa L. J Sea Res 44:55-64

O'Brien CM, Maxwell DL (2002) Towards an operational implementation of the precautionary approach within ICES-biomass reference points. Working document to the ICES study group on the further development of the precautionary approach to fisheries management CM2002/ACFM:10

Pérez-Rodríguez A, Morgan MJ, Saborido-Rey F (2009) Comparison of demographic and direct methods to calculate probabilistic maturation reaction norms for Flemish Cap cod (Gadus morhua). Evol Appl 2:291-298

Poos JJ, Brännström Å, Dieckmann U (2011) Harvestinduced maturation evolution under different life-history trade-offs and harvesting regimes. J Theor Biol 279: 102-112

Quandt RE (1958) The estimation of the parameters of a linear regression system obeying two separate regimes. J Am Stat Assoc 53:873-880

Quince C, Abrams PA, Shuter BJ, Lester NP (2008) Biphasic growth in fish. I. Theoretical foundations. J Theor Biol 254:197-206

Rijnsdorp AD (1993) Fisheries as a large scale experiment on life history evolution: disentangling phenotypic and genetic effects in changes in maturation and reproduction of North Sea plaice, Pleuronectes platessa L. Oecologia 96:391-401

Rijnsdorp AD, Storbeck F (1995) Determining the onset of

Editorial responsibility: Stylianos Somarakis, Heraklion, Greece sexual maturity from otoliths of individual female North Sea plaice, Pleuronectes platessa L. In: Secor DH, Dean JM, Campana SE (eds) Recent developments in fish otolith research. University of South Carolina Press, Columbia, SC, p 581-598

Rijnsdorp AD, Ibelings B (1989) Sexual dimorphism in the energetics of reproduction and growth of North Sea plaice, Pleuronectes platessa L. J Fish Biol 35:401-415

Rijnsdorp AD, van Damme CJD, Witthames PR (2010) Implications of fisheries-induced changes in stock structure and reproductive potential for stock recovery of a sexdimorphic species, North Sea plaice. ICES J Mar Sci 67 : 1931-1938

Roff DA (1991) The evolution of life-history variation in fishes, with particular reference to flatfishes. Neth J Sea Res 27:197-207

Salkeld PN (1995) Aspects of reproduction associated with the use of a segmented regression to describe the relationship between body weight and shell length of Mytilus edulis. Mar Ecol Prog Ser 124:117-128

Seber GAF, Wild CJ (2003) Nonlinear regression. John Wiley \& Sons, Hoboken, NJ

> Solmundsson J, Karlsson H, Palsson J (2003) Sexual differences in spawning behaviour and catchability of plaice (Pleuronectes platessa) west of Iceland. Fish Res 61: $57-71$

> Stamps JA, Mangel M, Philips JA (1998) A new look at relationships between size at maturity and asymptotic size. Am Nat 152:470-479

> Tsoularis A, Wallace J (2002) Analysis of logistic growth models. Math Biosci 179:21-55

van Walraven L, Mollet FM, van Damme CJG, Rijnsdorp AD (2010) Fisheries-induced evolution in growth, maturation and reproductive investment of the sexually dimorphic North Sea plaice (Pleuronectes platessa L.). J Sea Res 64: 85-93

Submitted: August 30, 2011; Accepted: December 20, 2011 Proofs received from author(s): March 14, 2012 